Annales de Parasitologie (Paris), t. 47, 1972, $\mathrm{n}^{\circ} 1$, pp. 51 à 75

\title{
Contribution à l'étude des Strigeata La Rue, 1926 (Trematoda : Strigeida) de Cuba
}

\author{
par G. DUBOIS et J. K. MACKO \\ Institut de Zoologie, Université de Neuchâtel, Suisse \\ Institutio Helminthologiae, Košice, Czechoslovakia
}

\begin{abstract}
Résumé
Parastrigea diovadena, Neodiplostomum centuri et $N$. isomegalocotyle sont décrits comme espèces nouvelles.

Actuellement, à Cuba, les Strigeata sont représentés par onze genres et vingt et une espèces. Cette étude confirme le caractère composite et la pauvreté relative de la faune helminthologique de cette île.
\end{abstract}

\section{Summary}

Parastrigea diovadena, Neodiplostomum centuri et $N$. isomegalocotyle are described as new species.

At the present time, there are eleven genus and twenty-one species of Strigeata in Cuba. The character composite and the relative poverty of the helminthofauna of this island is confirmed by the present study.

Une importante collection de Strigeata a été constituée par des chercheurs de la Division de Parasitologie de l'Académie cubaine des Sciences, à La Havane, et par des helminthologues de l'Institut parasitologique de Prague et de l'Institut helminthologique de Košice, dans l'Ile de Pinos et dans les Péninsules de Zapata et de Guanahacabibes. Elle compte 10 espèces de Strigeidae Railliet — dont une nouvelle — et 8 espèces de Diplostomidae Poirier - dont deux nouvelles.

Malheureusement, la plupart des matériels étaient dans un état bien médiocre, dû probablement à une fixation tardive. La macération a rendu le travail d'identification 
malaisé, et particulièrement délicat en ce qui concerne la description des espèces nouvelles.

Tous les matériels sont conservés au Muséum de l'Institut helminthologique de Košice.

Les indications relatives à la distribution géographique des espèces sont ordonnées suivant le protocole de dissection 1965-1966. Les numéros sont ceux des préparations.

\section{Familia STRIGEIDAE Railliet}

\section{Strigea falconis brasiliana Szidat, 1929}

Les quelques exemplaires récoltés à La Jaula, chez la Buse platyptère de Cuba, présentent les deux caractéristiques de la sous-espèce brasiliana, à savoir: la grosseur du cône génital (diamètre 240-320 $\mu$ ), qui est plus grand que l'ovaire, et la petitesse relative des œufs (89-99/55-60 $\mu$ ) (1), par rapport à ceux de la sous-espèce nominative.

Hôte: Buteo platypterus cubanensis Burns.

Distribution : Peninsula de Guanahacabibes, La Jaula (14-1-1966) [1821 b, $c, d]$.

Strigea vaginata (Brandes, 1888) Szidat, 1928

Syn. Gongylura vaginata (Brandes) Lutz, 1933;

Strigea ophiocystis Lutz, 1928 ;

Strigea ichthyocystis Lutz, 1929;

Strigea theriocystis Lutz, 1929 ;

Apatemon gracilis Caballero et Vogelsang, 1949, nec Rudolphi, 1819.

Ce parasite de Vulturidés est fréquent au Brésil et au Venezuela. Brandes (1888, 1890) puis Szidat (1929) l'ont décrit d'après des matériels recueillis par Natterer et conservés au Musée de Vienne : les hôtes sont Cathartes urubitinga Pelzeln et Sarcoramphus papa L. Dubois (1938), puis Caballero et Vogelsang (1949) le signalent chez Coragyps atratus (Bechst.). De nombreuses préparations de la collection A. Lutz proviennent de ce Vautour (of. Dubois 1970 b). Szidat (op. cit.) mentionne encore comme hôtes un Aigle huppé, Spizaetus ornatus (Daud.), et le Sériema ou Cariama cristata (L.) qui est un grand mangeur de Cobras aquatiques. (Ces Serpents hébergent les Tetracotyle de $« S$. ophiocystis $»)$.

Plusieurs matériels cubains proviennent de Cathartes a. aura (L.). La description suivante est basée sur le mieux conservé, bien que les exemplaires soient un peu aplatis $\left(n^{\circ} 1678 b\right)$.

LONGUEUR DES Vers: jusqu'à 3,2 mm. Segment antérieur ovoïde ou utriforme, 0,74-1,04/ 0,70-0,90 mm, recouvert d'épines cuticulaires; segment postérieur massif, cucumiforme et fortement arqué, 1,17-2,21/0,58-0,92 mm, séparé du précédent par une constriction assez mar$55 \mu$.

(1) D'après Pérez Vigueras (1944) : 67/45 $\mu$. D'après nos mesures sur son matériel : 68-91/42- 
quée, à extrémité caudale arrondie ou tronquée, sans délimitation de la bourse copulatrice. Rapport des longueurs: segment postérieur/segment antérieur $=1,5-2,3$. Ventouse buccale marginale, 110-160/100-130 $\mu$; pharynx largement ellipsoïdal, 105-145/90-145 $\mu$, subégal ou un peu plus petit, mais plus musculeux ; ventouse ventrale postéquatoriale ou équatoriale, 185 265/160-200 $\mu$. Glande protéolytique ovoïde, multilobulée, 190-270/210-370 $\mu$, située à la base du segment antérieur et flanquée ventralement, dans sa seconde moitié, de deux languettes virguliformes appliquées contre elle, longues de 105 à $190 \mu$, à pointe dirigée en arrière.

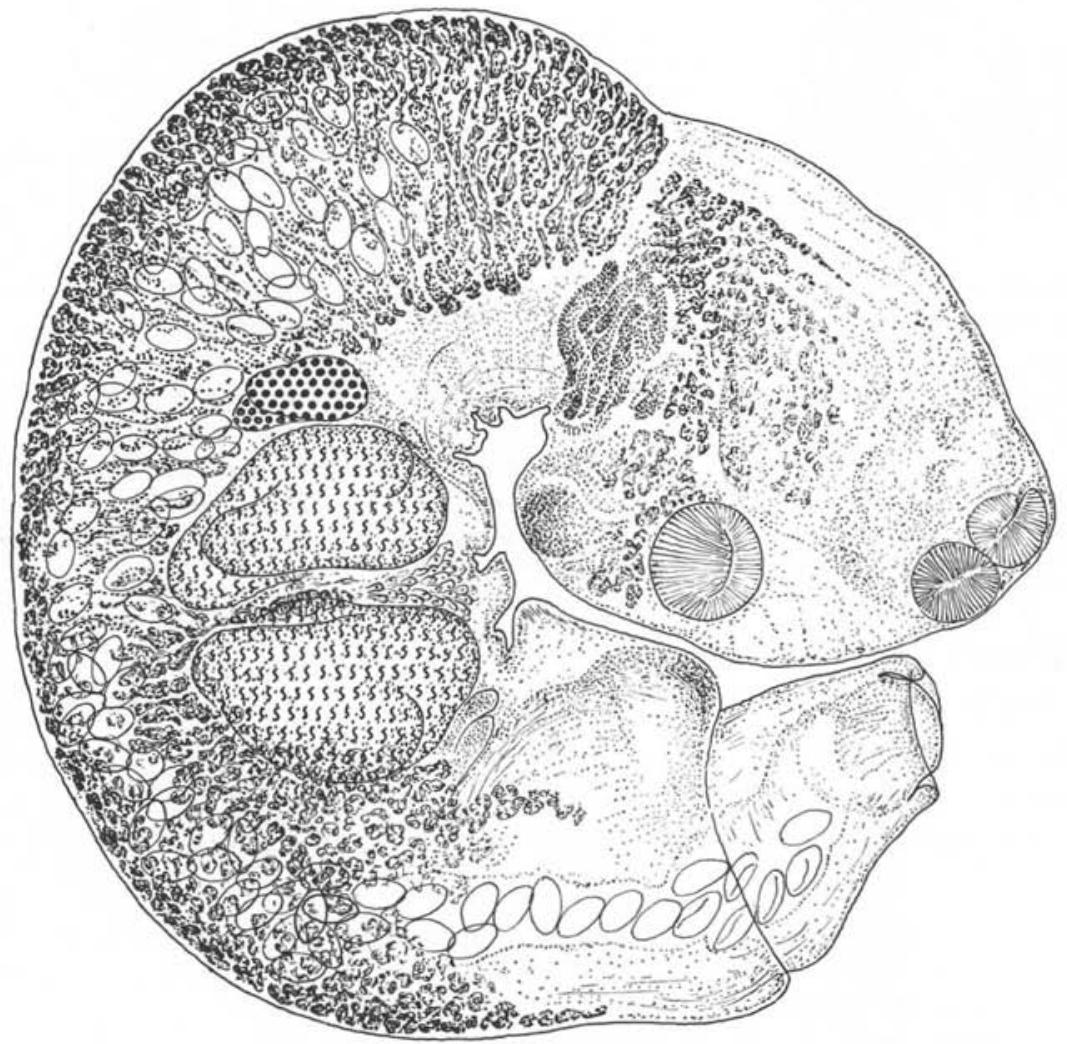

FIG. 1. - Strigea vaginata (Brandes), de Cathartes aura aura (L.) Longueur: $3,16 \mathrm{~mm}[1678 \mathrm{~b}]$

Ovaire réniforme, à hile ventral, 100-190/200-310 $\mu$, situé aux 28-34/100 du segment postérieur. Testicules bilobés, fortement recourbés en fer à cheval, à conoavité ventrale: le premier au contact de l'ovaire, 210-310/290-550 $\mu$, le second un peu plus grand, 220-400/350$570 \mu$. Vésicule séminale repliée sur elle-même, suivie d’un canal éjaculateur débouchant à l'entrée du cône génital. Vitellogènes à densité assez faible dans le segment antérieur; beaucoup plus abondants dans le segment postérieur, surtout dans la zone préovarienne, puis réduits à un ruban ventral qui se dilate en arrière des testicules, enveloppant en partie le cône génital 
et se terminant au niveau de l'anneau musculaire atrial. Bourse copulatrice volumineuse, occupant le dernier tiers du segment postérieur, à atrium peu profond ; cône génital énorme, 480$550 / 350-550 \mu$, atteignant jusqu'à $950 \mu$ de longueur en exsertion, traversé par les méandres ou les diverticules que le canal hermaphrodite développe à l'état de rétraction et dont les parois, épaisses de 15 à $25 \mu$, possèdent une musculature circulaire prépondérante. CEufs nombreux chez les exemplaires mûrs, 99-104/57-60 $\mu$.

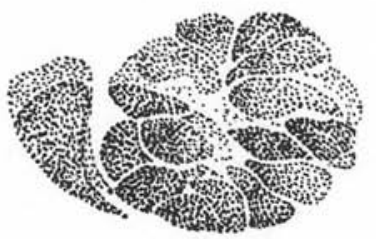

Fig. 2. - Strigea vaginata (Brandes), de Cathartes aura aura (L.). Glande protéolytique

Les exemplaires de la préparation $\mathrm{n}^{\circ} 1679 b$, pas trop mal conservés, mais fixés en extension et assez aplatis, mesurent 3,8 à $5,6 \mathrm{~mm}$ de longueur. Segment antérieur, 1,05-1,36/0,95$1,30 \mathrm{~mm}$; segment postérieur cylindrique, arqué dans sa première partie, 2,80-4,24/0,97$1,12 \mathrm{~mm}$. Rapport des longueurs : segment postérieur/segment antérieur $=2,5-3,1$. Ventouse buccale $135-170 / 100-150 \mu$; pharynx 130-150/115-135 $\mu$; ventouse ventrale 250-280/180$240 \mu$. Ovaire 170-230/290-380 $\mu$, situé aux 26-37/100 du segment postérieur. Testicule antérieur 430-500/530-750 $\mu$; testicule postérieur 400-500/600-710 $\mu$. (Selon leur diamètre transversal, les trois gonades mesurent respectivement $500 \mu, 800 \mu$ et $880 \mu$ ). Cône génital

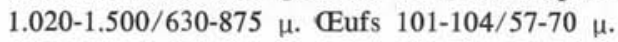

\section{HôTES : Cathartes aura aura (L.).}

Distribution: Isla de Pinos, Rancho del Tesoro (11-X-1965) [1464 e, $f, i$; $1468 d]$.

Peninsula de Zapata, Canal de Zapata (6-XII-1965) [1678 b, 1679 b].

Peninsula de Guanahacabibes, Reservación (21-I-1966) [1901 $a, b, c ; 1902 a, b]$.

\section{Apharyngostrigea cornu (Zeder, 1800), Ciurea, 1927}

Syn. Strigea ardearum ex parte Lutz, 1928 (cf. Dubois 1970 b) ; Apharyngostrigea bilobata Olsen, 1940.

En Amérique du Nord, cette espèce a été signalée par O. W. Olsen (1940: Minnesota), Byrd et Ward (1943 : Mississippi, Géorgie et Tennessee), Dubois (1958: Californie ; 1967 : Canada ; 1969 : Massachusetts). Les hôtes sont divers Ardéidés.

Au Brésil et au Venezuela, Lutz (1928) la désignait sous le nom de «Strigea ardearum»: c'est une espèce commune des Hérons diurnes et nocturnes, que nous avons identifiée (1970 b, pp. 169-170) avec $A$. cornu (ex parte).

A Cuba, Pérez Vigueras (1944) l'a retrouvée assez fréquemment chez Ardea herodias L. et Nycticorax nycticorax hoactli (Gm.). 
L'espèce est caractérisée par son segment antérieur ovoïde ou bulbiforme, nettement distinct du segment postérieur cylindrique, arqué dans sa première moitié, peu à peu atténué dans la seconde, avec bourse copulatrice légèrement délimitée ; par une glande protéolytique arrondie ou ovoïde, intersegmentaire, parfois refoulée tout au début du segment postérieur; par l'ovaire réniforme et des testicules multilobés; par des vitellogènes profus dans le segment antérieur, où ils pénètrent plus ou moins en avant dans les deux lèvres de l'organe tribocytique et remontent dans la paroi dorsolatérale du corps jusqu'au niveau de la ventouse buccale ; plus clairsemés à la jonction intersegmentaire, puis densément accumulés jusqu'à l'ovaire, au-delà duquel ils se limitent à un ruban ventral médian qui se dilate en arrière des testicules sous forme d'un manchon dans les parois de la bourse copulatrice.

Les mesures prises sur les matériels cubains (n ${ }^{\circ s} 1643 c$ et $1673 d$; coll. Pérez Vigueras, $\mathrm{n}^{\text {os }} 38,60$ et 77 ) sont les suivantes :

LONGUEUR DU VER: jusqu'à $4,75 \mathrm{~mm}$. Ventouse buccale $110-190 / 160-235 \mu$; ventouse ventrale 230-250/230-265 $\mu$. Glande protéolytique 180-320/210-330 $\mu$, flanquée d'une «languette » ventro-antérieure mesurant $135-170 / 50-70 \mu$. Situation de l'ovaire aux 27-41/100 du segment postérieur (moyenne $35 / 100$ ). CEufs très nombreux à maturité complète, 87-100/57$68 \mu$.

Hôtes: Butorides virescens maculatus (Bodd.) $=$ B. brunescens et Ardea herodias $\mathrm{L}$.

Distribution: Peninsula de Zapata, St-Tomas, Canal de Zapata (1-XII-1965) [1643c] ; Salinas, Canal de Zapata (4-XII-1965) [1673d].

Apharyngostrigea multiovata (Vigueras, 1944) Dubois et Vigueras, 1949

Syn. Strigea ardearum ex parte Lutz, 1928 (cf. Dubois 1970 b) ;

Ophiosoma multiovatum Pérez Viguéras, 1944 ;

Apharyngostrigea insulae Pérez Vigueras, 1944 ;

Apharyngostrigea gundlachi Pérez Vigueras, 1944 ;

Apharyngostrigea cornu Cable, Connor et Balling, 1960, nec Zeder.

Pérez Vigueras (1944) a attribué à des genres différents les deux stades, pauciovigène $(A$. insulae) et pluriovigène $(O$. multiovatum), de la maturation d'une même espèce que, dans une note rectificative publiée en commun (1949), nous avons appelée Apharyngostrigea multiovata. De plus, cet auteur a décrit assez sommairement une Apharyngostrigea gundlachi, de Ixobrychus exilis (Gm.), que Dubois (1968 b) a d'abord rapportée à $A$. pipientis (Faust), mais qui, en raison de l'aspect ovoïde ou cucumiforme de la glande protéolytique multilobulée $(320 / 200 \mu)$ et de la grosseur relative du cône génital (220-225/190-220 $\mu)$, s'identifie mieux à $A$. multiovata dont elle représente une forme jeune, ovigère. Contrairement aux observations de Pérez Vigueras, les vitellogènes, limités ventralement dans la zone testiculaire, se prolongent 
jusqu'à l'extrémité du corps, où ils forment un manchon dans les parois de la bourse copulatrice.

Cable, Connor et Balling (1960) ont redécrit cette espèce sous le nom d'Apharyngostrigea cornu, d'après deux spécimens ovigères ( Eggs relatively few in number») provenant d'une Florida caerulea (L.) de Porto Rico. Dubois (1967) en a publié une nouvelle description d'après quatre exemplaires (à œufs nombreux) récoltés par Robert-L. Rausch dans le même hôte, en Ohio. Enfin, dans la collection Elisabeth-M. Boyd se trouvaient trois spécimens recueillis au Massachusetts dans l'intestin d'une Ardea herodias L.

Nous avons retrouvé $(1970 b)$ A. multiovata dans la collection A. Lutz; elle était hébergée par Egretta alba egretta (Gm.) et par Butorides striatus (L.).

L'espèce est caractérisée par son corps allongé, à segment antérieur infundibuliforme (avec ventouse ventrale pré ou sub-équatoriale), à segment postérieur longuement claviforme, cylindrique jusqu'au niveau de l'ovaire, puis légèrement renflé dans la zone testiculaire (avec bourse copulatrice très faiblement délimitée et à peine plus étroite), par un cône génital relativement gros (320-400/265-330 $\mu$ ), traversé par un canal hermaphrodite dont la paroi est pourvue d'une forte musculature circulaire (2). La glande protéolytique est ovoïde à cucumiforme (360-475/170-210 $\mu$ ), flanquée ventralement, au niveau de sa première moitié, de deux petites masses allongées, en forme de languette appointie en arrière. L'ovaire réniforme et fortement recourbé se situe à mi-longueur du segment postérieur ou plus en avant. Les testicules sont multilobés, très développés et contigus. Les vitellogènes paraissent peu abondants dans le segment antérieur, même chez les individus à maturité complète : ils ne pénètrent que dans les lèvres de l'organe tribocytique jusqu'à la hauteur de la ventouse ventrale et, de celle-ci, dans la paroi dorso-latérale du corps jusqu’à la ventouse buccale, souvent avec une densité moindre; ils se raréfient au niveau de la glande protéolytique, puis envahissent la première moitié du segment postérieur, se réduisant sous les testicules à un ruban ventral qui se dilate au-delà pour former un manchon de follicules dans les parois de la bourse copulatrice et atteindre l'extrémité du corps.

Les mesures prises sur les préparations les moins altérées sont les suivantes (voir tableau ci-contre):

Hôtes : Florida caerulea (L.), Egretta alba (L.), Egretta alba egretta (Gm.), Hydranassa tricolor ruficollis (Gosse), Leucophoyx thula (Molina), Butorides virescens maculatus (Bodd.), Ajaia ajaja (L.) (exempl. jeunes) (3).

Distribution: Isla de Pinos, Sierra Cubitas (26-X-1965) [1480 $a, b, d]$; Canal de Vigil, La Gloria (28-X-1965) [1490 d, e] ; Laguna Grande, La Gloria (28-X-1965) $[1517 b, c]$.

(2) Ce cône était bien visible, tel que Pérez Vigueras l'a dessiné (1944, fig. 4), avant l'écrasement accidentel de la préparation de $A$. insulae.

(3) Un exemplaire provenant d'Ardeola ibis (L.) ( ${ }^{\circ} 1504 a$, Canal de Vigil, La Gloria) est probablement identifiable avec Apharyngostrigea multiovata. 


\begin{tabular}{|c|c|c|c|}
\hline & $\mathrm{N}^{o s} 1480 a, b, d$ & $\mathrm{~N}^{o s} 1683 e, f, g$ & $\mathrm{~N}^{*} 1639 c$ \\
\hline Long du Ver ... & jusqu’à 5,2 mm & jusqu’à 4,6mm & jusqu'à 7 mm \\
\hline Segm. antér. .... & $0,85-1,20 / 0,53-0,69$ & $(1,50-1,75 / 0,77-0,91)^{\text {* }}$ & $1,25-1,50 / 0,80-0,90$ \\
\hline Segm. postér. ... & $3,15-4,45 / 0,42-0,60$ & $2,80-3,00 / 0,40-0,56$ & $4,55-5,70 / 0,66-0,70$ \\
\hline Vent. bucale .... & $73-95 / 81-104 \mu$ & $90-155 / 110-160 \mu$ & $120 / 150 \mu$ \\
\hline Vent. ventrale .. & $150-155 / 190-225$ & $125-200 / 200-230$ & $160 / 230$ \\
\hline G1. protéol. .... & $380-470 /(240-265)^{* *}$ & $310-360 / 180-220$ & $420 / 250$ \\
\hline Ovaire $\ldots \ldots \ldots$ & $170-250 / 255-330$ & $170-210 / 240-400$ & $230-240 / 300-370$ \\
\hline Test. antér. .... & $420-540 / 400-480$ & $420-540 / 340-415$ & $410-720 / 540-560$ \\
\hline Test. postér. ... & $420-640 / 370-480$ & $450-600 / 340-450$ & $590-740 / 600-630$ \\
\hline Cône génital ... & $340-400 / 320-380$ & - & $450-480 / 450$ \\
\hline Eufs $\ldots \ldots \ldots$. & $86-104 / 63-70^{* * * *}$ & $84-89 / 54-62$ & $99-104 / 58-74$ \\
\hline
\end{tabular}

(*) Exemplaires assez fortement macérés et aplatis.

(**) Chez tous les exemplaires cette glande est déformée et surtout élargie par l'aplatissement des préparations.

(***) Exemplaires jeunes. Les ceufs paraissent quelque peu dilatés par l'aplatissement. D'après Pérez Vigueras, ils mesurent 88-95/50-67 $\mu$; d'après Cable et al., 95-97/59-61 $\mu$; d'après Dubois (coll. Rausch), 89-105/60-70 $\mu$.

Peninsula de Zapata, St. Tomas, Canal de Zapata (1-XII-1965) [1639 c, $1640 c$ ], (6-XII-1965) [1683 $c, e, f, g]$; Playa Larga, Canal de Zapata (4-XII-1965) [1669 b] ; Salinas, Canal de Zapata (8-XII-1965) [1723d, juv.].

Peninsula de Guanahacabibes, La Florida (14-I-1966) [1806a] ; Reservación (27-I-1966) [1934 $a, b]$.

Parastrigea cincta (Brandes, 1888) Szidat, 1928

Les Vers, un peu aplatis, atteignent jusqu'à $5 \mathrm{~mm}$ de longueur. Le segment antérieur est piriforme: son aspect résulte du grand développement des expansions latérales de la lèvre dorsale de l'organe tribocytique. Les ventouses sont petites et faibles: la buccale, souvent rétractée, ne mesure que 80-110/115-130 $\mu$; la ventrale est à peine plus grande, 135-150/145-165 $\mu$; elle se situe entre les extrémités céphaliques des deux expansions latérales. Le pharynx a comme dimensions 95-120/80-110 $\mu$.

Le segment postérieur est sacciforme, à largeur maximum dans la zone testiculaire. La bourse copulatrice, large, mais peu profonde, à grande ouverture terminale, apparaît plus ou moins délimitée. Les testicules sont volumineux, multilobés et contigus. L'ovaire réniforme se situe dans le premier cinquième du segment postérieur. Quant aux follicules vitellogènes, ils s'étendent en avant jusqu'au niveau de la ventouse ventrale, s'accumulant dans les deux expansions latérales de l'organe tribocytique; ils sont encore largement répartis à la base du segment antérieur et au début du segment postérieur, en avant de l'ovaire et jusqu'à la face dorsale du corps, puis confinés ventralement dans la zone testiculaire sous forme d'une traînée assez large et dense, attei- 
gnant la bourse copulatrice, au-devant de laquelle ils remontent latéralement pour pénétrer dans ses parois. Les œufs nombreux mesurent 105-112/60-70 $\mu$.

Hôte : Ajaia ajaja (L.).

Distribution: Peninsula de Zapata, Salinas, Canal de Zapata (8-XII-1965) $[1724 b, f, g]$.

RemARQUe: L'exemplaire représenté par Szidat (1929, fig. 16) est de plus grande taille (jusqu'à $7 \mathrm{~mm}$ ); il a atteint une maturité sexuelle plus complète et contient beaucoup plus d'œufs. La réduction des vitellogènes dans la partie postérieure de son corps paraît corrélative au développement des testicules et à cette surproduction d'œufs. Ceux-ci sont plus petits (80/ $50 \mu$ ), mais ceux de $P$. cincta conservé dans la collection A. Lutz (cf. Dubois 1970 b, p. 171) mesurent $89-120 / 52-68 \mu$.

Nous croyons pouvoir attribuer à $P$. cincta le matériel recueilli chez un Falco sparverius L. (4). Les exemplaires qui mesurent 6,2 à $7,5 \mathrm{~mm}$ ne sont qu'au début de leur maturité : les œufs sont peu nombreux $(99-110 / 61-70 \mu)$. Les testicules bilobés, à lobes lobulés, sont allongés transversalement et ne présentent qu'un développement médiocre : le premier 560-770/ 1.470-1.570 $\mu$, le second 800-875/1.300-1.570 $\mu$. L'ovaire réniforme, 450-660/770-950 $\mu$, qui n'est pas refoulé par eux, se situe aux 41-45/100 du segment postérieur. Ce dernier n'est pas dilaté par les gonades : il apparaît subcylindrique à longuement sacciforme ; sa largeur maximum se mesure au-devant de l'ovaire. La glande protéolytique est ovoïde, 290-310/145-165 $\mu$; elle se situe aux 58-65/100 du segment antérieur qui est typiquement bulbiforme, toujours plus large que le reste du corps. Les expansions latérales de l'organe tribocytique, où s'accumulent les vitellogènes, mesurent $1.120-1.400 / 460-750 \mu$; presque contiguës en avant, elles divergent fortement en arrière.

Hôte: Falco sparverius sparverius L.

Distribution: Isla de Pinos, Canal de Vigil, La Gloria (28-X-1965) [1494 $b$, $d, e]$.

Parastrigea diovadena $\mathrm{n}$. sp.

Plusieurs exemplaires de ce parasite (malheureusement assez mal conservés) ont été récoltés dans un Ibis blanc, Eudocimus albus (L.), à St-Tomas.

Diagnose. Longeur des Vers : 2-3,7 mm. Segment antérieur oblong, profondément utriforme à largement piriforme, 0,80-1,47/0,66-1,13 mm, dans lequel les deux expansions latérales de la lèvre dorsale de l'organe tribocytique - mesurant chacune 370-830/230-400 $\mu$ et qui s'avancent jusqu'au niveau acétabulaire - se signalent par une forte concentration des vitellogènes, mais ne déterminent pas de saillies à l'extérieur; segment postérieur sacciforme ou conoïdal, tronqué en arrière, $1,29-2,24 / 0,70-1,10 \mathrm{~mm}$, délimité du précédent par une constriction, à large ouverture bursale. Rapport des longueurs : segment postérieur/segment antérieur $=1,4-1,8$. Ventouse buccale submarginale, $70-100 / 95-110 \mu$, suivie d'un pharynx très difficile-

(4) Dans la collection A. Lutz, nous avons retrouvé $P$. cincta chez un Vautour (vulgo "gavião») et chez Micrastur ruficollis gilvicollis (Vieill.) (longueur : jusqu'à $7 \mathrm{~mm}$ ). 
ment visible, mesurant 42-55 $\mu$ de diamètre ; ventouse ventrale rapprochée des précédents, 115 150/115-160 $\mu$, située aux 32-41/100 de la longueur du segment. Une glande protéolytique, aussi grosse que l'ovaire et qui paraît très caractéristique de l'espèce, se présente sous la forme de deux lobes ovoïdes, mesurant chacun 180-200/240-300 $\mu$, situés dorsalement et obliquement à la base du segment antérieur.

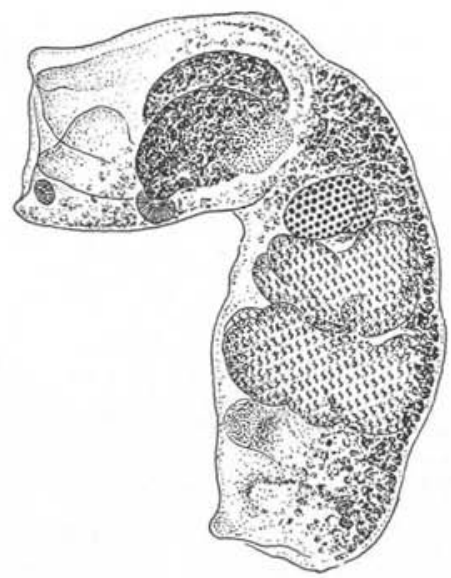

FIG. 3. - Parastrigea diovadena n. sp., de Eudocimus albus (L.). Longueur : $2,82 \mathrm{~mm}$ [1641 $h]$

Ovaire réniforme, $170-320 / 230-450 \mu$, à hile ventral ou postérieur, situé au $1 / 5 \mathrm{du}$ second segment (16-23/100). Testicules très développés, grossièrement lobés et subégaux : le premier 380-420/670-820 $\mu$, le deuxième 400-450/690-870 $\mu$. Vitellogènes concentrés dans les deux expansions latérales de l'organe tribocytique, pénétrant aussi dans les parois du segment antérieur jusqu'au niveau de la ventouse ventrale, au-delà duquel ils ne s'avancent que dorsolatéralement en se raréfiant; abondants dans la zone préovarienne, puis limités à un large ruban ventral qui se dilate en arrière des testicules pour pénétrer dans les parois de la bourse copulatrice, jusqu'à l'extrémité du corps. CEufs nombreux, 90-100/62-68 $\mu$.

Hôte: Eudocimus albus (L.).

Distribution: Peninsula de Zapata, St-Tomas, Canal de Zapata (1-XIl-1965) $[1641 f, g, h, i, j]$.

\section{HoLOTYPE : $1641 h$.}

Parastrigea diovadena est donc bien caractérisée par la forme et la situation de la glande protéolytique. Cette particularité mise à part, elle se rapprocherait de $P$. caballeroi Dubois, qui a été trouvée au Venezuela chez un Jabiru mycteria (Licht.), mais s'en distingue par ses plus petites dimensions, par l'absence de saillies au niveau des expansions latérales et par une occupation plus complète du segment postérieur par les testicules. 


\section{Parastrigea mexicana Coil, 1957}

L'espèce a été retrouvée dans un Récurvirostridé, Himantopus himantopus mexicanus (Müller), sous sa forme typique, mais avec des dimensions plus grandes (5). Coil l'avait décrite d'après des spécimens provenant d'une Avocette, Recurvirostra americana Gm., du Mexique.

LONGUEUR DES Vers : 4,9-5 mm. Segment antérieur largement piriforme, 1,92-2,10/2,80$2,90 \mathrm{~mm}$, avec expansions latérales ovoïdes très développées et saillantes, contiguës sur la ligne médiane ; segment postérieur ovoïdo-conique, 3-3,1/1,92-2 mm. Rapport des longueurs: segment postérieur/segment antérieur $=1,48-1,60$. Ventouses et pharynx indistincts.

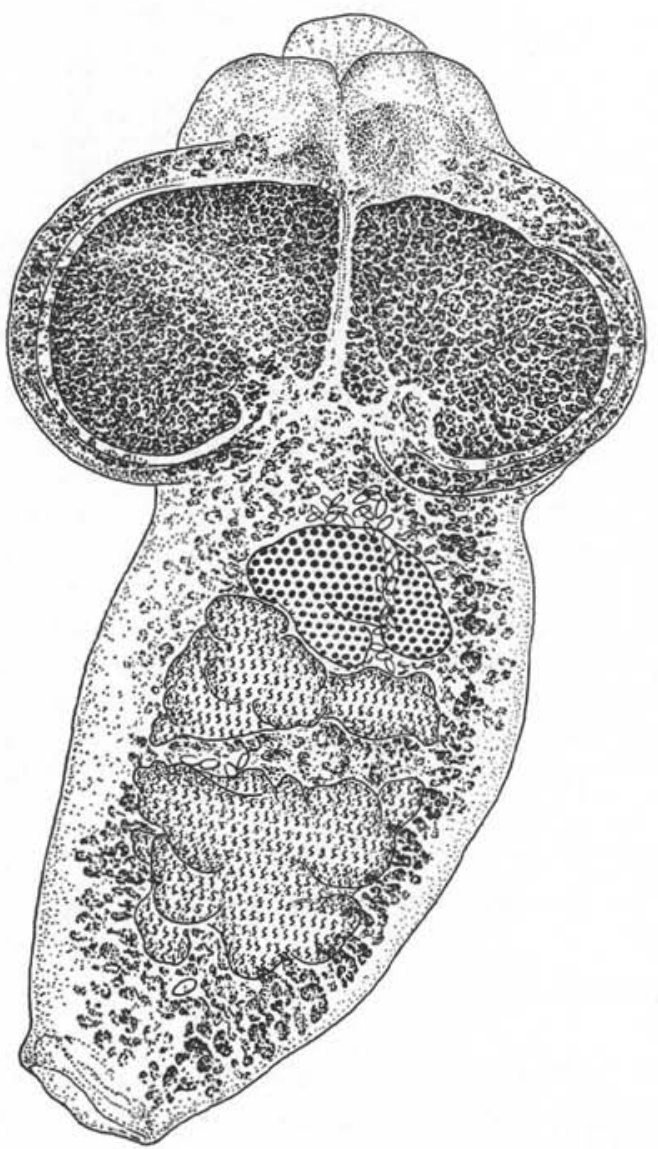

FIG. 4, - Parastrigea mexicana Coil, de Himantopus himantopus mexicanus (Müller). Longueur: $5 \mathrm{~mm}$, vue ventrale $\left[\begin{array}{lll}1531 & d\end{array}\right]$

(5) Les préparations paraissent aplaties. 
Complexe des gonades ovalaire, faisant figure de «noyau» dans le segment postérieur. Ovaire réniforme, 420-500/875-900 $\mu$, situé aux 14-18/100 de ce segment. Testicules multilobés, dont la masse mesure 1.500-1.750/1.300-1.400 $\mu$. Vitellogènes concentrés dans les expansions latérales du segment antérieur, remontant aussi dans ses parois; moins denses à la face ventrale du segment postérieur, dont ils atteignent à peu près l'extrémité caudale. Bourse copulatrice peu profonde, largement ouverte. Eufs grands, nombreux, normalement allongés, $105-125 / 65-72 \mu$.

HôTE: Himantopus himantopus mexicanus (P. L. S. Müller).

Distribution: Isla de Pinos, Playa Pilota, Camagüey (30-X-1965) [1531 $d, e]$.

\section{Apatemon sp.}

Une préparation contient trois spécimens d'un Apatemon (sous-genre et espèce indéterminables).

Hôte : Aix sponsa (L.).

Distribution: Isla de Pinos, Canal de Vigil, La Gloria (28-X-1965) [1496 a].

\section{Cardiocephaloides medioconiger}

(Dubois et Vigueras, 1949) Baer, 1969

Syn. Strigea bursigera Linton, 1928, nec Brandes, 1888 ;

Cardiocephalus brandesi Pérez Vigueras, 1944, nec Szidat, 1928 ;

Cardiocephalus medioconiger Dubois et Vigueras, 1949 ;

Cardiocephalus megaloconus Cable, Connor et Balling, 1960.

Cette espèce, parasite de Laridés (Larus, Sterna et Rhynchops), est bien caractérisée par les dimensions moyennes de la bourse copulatrice et par la présence d'un ductus ejaculatorius très musculeux, situé dorsalement et dont le diamètre varie entre 100 et $260 \mu$, les parois ayant 20 à $40 \mu$ d'épaisseur sur les matériels cubains. Elle a une distribution géographique étendue, partant des Antilles et de l'Amérique centrale (Panama) pour longer le golfe du Mexique et toute la côte orientale des Etats-Unis.

Les exemplaires recueillis à Cuba mesurent jusqu'à $14 \mathrm{~mm}$ de longueur. Leurs œufs, très nombreux, ont 99-120/63-80 $\mu$ (épaisseur de la coque : 3-4 $\mu$ ).

Hôte: Thalasseus maximus (Bodd.).

Distribution: Isla de Pinos, Playa Pilota, Camagüey (30-X-1965) [1532 v, 1-3, $1533 d, e, f]$.

\section{Cotylurus (Cotylurus) gallinulae gallinulae (Lutz, 1928) Dubois, 1937}

Syn. Choanodiplostomum lintoni Pérez Vigueras, 1944.

Lutz (1928) a observé ce parasite chez des «gallitos de agua», tant au Brésil qu'au Venezuela (6). Il l'a attribué au genre Strigea Abildg. Pérez Vigueras (1944) en

(6) Dans la collection A. Lutz, nous avons trouvé (1970 b) 3 exemplaires provenant de Gallinula chloropus galeata (Licht.): le lectotype a été déposé à l'Université de Neuchâtel (collection G. Dubois, $\mathrm{n}^{\circ} \mathrm{F}$ 1), les deux autres (paralectotypes) sont conservés à l'Institut Oswaldo Cruz, Rio de J. $\left(\mathrm{N}^{\circ} 25815\right.$ et 30469$)$. 
a récolté un spécimen chez Gallinula chloropus cerceris Bangs (cf. Dubois, 1968 , fig. 211). Un autre exemplaire a été retrouvé à Cuba chez ce dernier hôte : il est bien caractérisé par son habitus et par la grosseur des pseudo-ventouses ovoïdes (qui apparaissent ici bourrées de noyaux cellulaires). En voici les mesures (préparation un peu aplatie) :

LONGUEUR DU Ver : 2,4 mm. Segment antérieur 0,95/1,20 mm ; segment postérieur 1,45/ $0,96 \mathrm{~mm}$. Rapport des longueurs : segment postérieur/segment antérieur $=1,53$. Ventouse buccale $150 / 145 \mu$; pharynx petit $68 / 60 \mu$; ventouse ventrale $210 \mu$; pseudo-ventouses 330/ $200 \mu$. Ovaire réniforme, $140 / 225 \mu$ situé aux $15 / 100$ du segment postérieur. Testicule antérieur $200 / 400 \mu$ (diamètre dorso-ventral) ; testicule postérieur $245 / 380 \mu$. Formation musculaire ovoïde du bulbe génital 47/34 $\mu$. CEufs rares 85-102/58-63 $\mu$.

HôTE: Gallinula chloropus cerceris Bangs.

Distribution: Peninsula de Zapata, Caleta el S. «Plan Fidel », Zapata (7-XII1965) $[1704 f]$.

\section{Tetracotyle sp.}

Deux préparations contiennent chacune un Tetracotyle (indéterminable).

Hôte : Quiscalus niger caribaeus (Todd).

Distribution: Isla de Pinos, Cayo Piedra (8-X-1965) [1435 c, f].

Familia DIPLOSTOMIDAE Poirier

\section{Diplostomum (Austrodiplostomum) compactum}

(Lutz, 1928) Dubois, 1970

Syn. Alaria compacta Lutz, 1928 ;

Hysteromorpha compacta (Lutz) Dubois, 1937 ;

Austrodiplostomum mordax Szidat et Nani, 1951.

L'espèce a été retrouvée dans deux Cormorans. Elle paraît donc bien inféodée à ce genre d'Oiseaux : Szidat et Nani (1951) mentionnaient plusieurs cas d'infestation de l'hôte-type, Phalacrocorax olivaceus olivaceus (Humb.), au Rio de la Plata. Les spécimens de Cuba permettent d'en compléter la description:

Corps elliptique, linguiforme, fortement atténué en arrière, long de 2,1 à 2,6 mm, large de 1 à $1,3 \mathrm{~mm}$, à segment antérieur concave ventralement et muni de profondes pseudoventouses cupuliformes, 170-210/140-160 $\mu$, dont les ouvertures sont disposées obliquement et en dedans, à la hauteur du pharynx ; à segment postérieur peu développé et largement conique. Rapport des longueurs: segment postérieur/segment antérieur $=0,40-0,45$. Ventouse buccale 75-105/65-85 $\mu$; pharynx à peine plus petit, mais plus musculeux, 73-94/57-80 $\mu$; ventouse ventrale absente; organe tribocytique grand, 520-740/470-600 $\mu$, de contour ovale à subtriangulaire. 


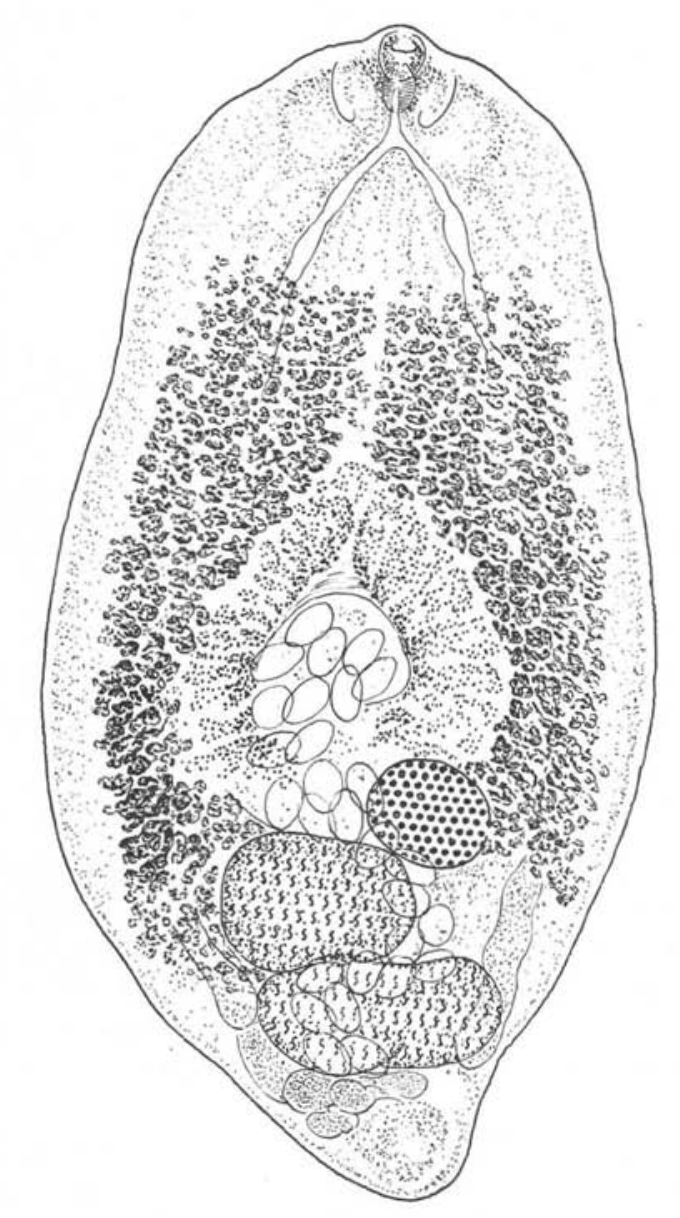

F1G. 5. - Diplostomum (Austrodiplostomum) compactum (Lutz), de Phalacrocorax auritus floridanus (Audub.). Longueur: $2,13 \mathrm{~mm}$, vue ventrale $[1604 a]$

Ovaire ellipsoïdal, 165-180/215-230 $\mu$, submédian, situé à la limite intersegmentaire, opposé obliquement ou antérieur au premier testicule qui est ovoïde, asymétriquement développé, 190-250/265-360 $\mu$; testicule postérieur bilobé, allongé tranversalement, 160-210/310$440 \mu$. Vitellogènes à petits follicules largement distribués dans le segment antérieur, où ils entourent l'organe tribocytique et s'avancent jusqu'au $1 / 3$ de cette partie du corps ; pénétrant latéralement dans le segment postérieur jusqu'au niveau intertesticulaire. Bourse copulatrice petite, abritant un cône génital (diamètre 50-60 $\mu$ ). CEufs nombreux (jusqu'à une septantaine), 85-100/55-65 $\mu$. 
Hôtes: Phalacrocorax olivaceus mexicanus (Brandt) (= brasilianus mexicanus) et $P$. auritus floridanus (Audub.).

Distribution: Isla de Pinos, Playa Pilota, Camagüey (30-X-1965) [1537d].

Peninsula de Zapata, Salinas, Canal de Zapata (1-XII-1965) [1604a].

\section{Diplostomum (Tylodelphys) elongatum (Lutz, 1928) Dubois, 1961}

Syn. Alaria elongata Lutz, 1928 ;

Diplostomum brevisegmentatum Pérez Vigueras, 1944.

Deux exemplaires fortement macérés et plus ou moins aplatis, l'un non ovigère, l'autre ovigère, ont été récoltés chez deux Grèbes.

LONGUEUR DES VERS : $1,82 \mathrm{~mm}$ et $2,35 \mathrm{~mm}$ (7). Les pseudo-ventouses mesurent respectivement $135 / 70-85 \mu$ et $210 / 105 \mu$. Ces dimensions sont bien caractéristiques de l'espèce (8).

Hôtes: Podiceps dominicus dominicus (L.) et Podilymbus podiceps podiceps (L.).

Distribution: Isla de Pinos, Laguna Grande, La Gloria (28-X-1965) [1523 m]. Peninsula de Zapata, Caleta el S. «Plan Fidel », Zapata (7-XII-1965) [1709 a].

\section{Diplostomum sp.}

Un seul exemplaire, assez médiocrement conservé, figurait parmi quelques spécimens de Posthodiplostomum nanum et un individu de $P$. macrocotyle provenant d'une Egratte blanche. Il ressemble au Diplostomum (Dolichorchis) mashonense Beverley-Burton, 1963, mais s'en distingue par la situation de l'organe tribocytique en arrière de la mi-longueur du corps, par de plus longues pseudo-ventouses et par le fait que la ventouse ventrale est nettement plus grande que la ventouse buccale.

Corps linguiforme, morphologiquement indivis, long de $1,19 \mathrm{~mm}$. Segment antérieur longuement elliptique, $0,87 / 0,40 \mathrm{~mm}$; segment postérieur courtement ovoïde, $0,32 / 0,28 \mathrm{~mm}$, à peine distinct du précédent, avec bourse copulatrice très légèrement délimitée. Rapport des longueurs : segment postérieur/segment antérieur $=0,37$. Ventouse buccale $57 / 65 \mu$; pharynx large de $38 \mu$; ventouse ventrale $84 / 94 \mu$; organe tribocytique $145 / 145 \mu$; pseudo-ventouses $120 / 50 \mu$ (Les glandes génitales sont peu distinctes).

Follicules vitellogènes surtout concentrés autour de l'organe tribocytique, plus clairsemés en avant de la ventouse ventrale ; à densité un peu plus faible dans la première moitié du segment postérieur, mais avec une forte condensation bilatérale entre les testicules et la bourse copulatrice qui reste à découvert.

Situation dans le segment antérieur: limite frontale des vitellogènes aux 55/100; ventouse ventrale aux 72/100; bord frontal de l'organe tribocytique aux 83/100.

(7) Lutz indique 1,5 à $2,2 \mathrm{~mm}$.

(8) Les pseudo-ventouses de $D$. (T.) americanum Dubois [espèce voisine du Brésil et du Venezuela, parasite de Mycteria americana L. et de Jabiru mycteria (Licht.)] ne mesurent que 50-122/ $68-80 \mu$. 
Hôte : Egretta alba (L.).

Distribution: Peninsula de Zapata, St. Tomas, Canal de Zapata (2-XII-1965) $[1633 \mathrm{~b}]$.

\section{Neodiplostomum centuri n. $\mathrm{sp}$.}

Plusieurs spécimens de ce parasite ont été trouvés chez des Picidés du genre Melanerpes Swainson, 1832 (syn. Centurus Swainson, 1837). Deux exemplaires furent encore recueillis chez un Iguanidé, Anolis equestris kosleri (sic), au même endroit (Rancho del Tesoro) et à la même date (11-X-1965) que les lots précédents ( $\mathrm{n}^{\circ} 1463$ ).

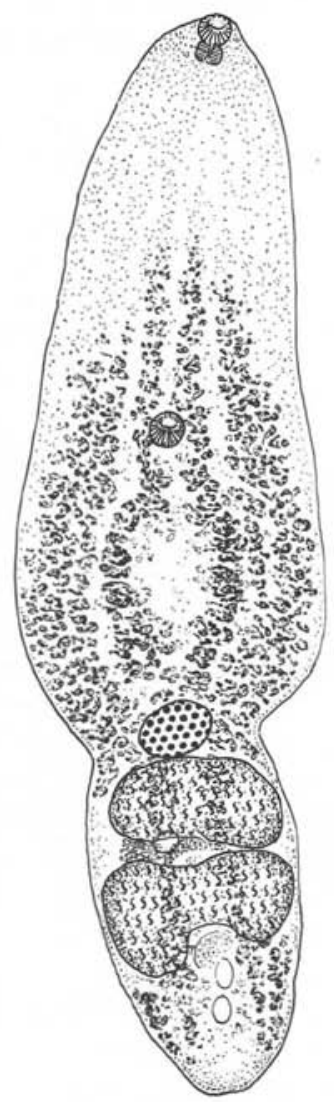

F1G. 6. - Neodiplostomum centuri n. sp., de Melanerpes superciliaris murcens (Bangs). Holotype. Longueur : $4 \mathrm{~mm}$, vue dorsale (Le pharynx est dilaté.) [1463 c] 
Diagnose. Longueur des Vers : 2-4,4 mm (9). Segment antérieur lancéolé, 1,42-3,05/0,58$1,29 \mathrm{~mm}$; segment postérieur ovoïde, 0,58-1,33/0,52-0,82 mm. Rapport des longueurs : segment postérieur/segment antérieur $=0,38-0,55$ (moy. 0,46). Ventouse buccale 70-75/84-102 $\mu$; prépharynx très court, 15-20 $\mu$; pharynx ellipsoïdal, musculeux, 65-75/44-47 $\mu$; œsophage court, $30 \mu$; ventouse ventrale 73-86/80-105 $\mu$, située à mi-longueur du segment (plus en arrière par étirement du Ver) ; organe tribocytique circulaire (à légèrement elliptique), 370480/360-450 $\mu$; glande protéolytique bilobée, au niveau de son bord postérieur.

Ovaire ovoïde, 130-190/180-320 $\mu$, situé à la limite intersegmentaire. Testicules massifs, occupant la plus grande partie du segment postérieur: le premier légèrement asymétrique, réniforme ou même cunéiforme, à convexité antérieure, 240-370/390-680 $\mu$, le second typiquement bilobé, à lobes développés caudalement, 245-410/425-760 $\mu$. Vitellogènes pénétrant largement dans le segment antérieur, s'avançant sous forme de six traînées et en se raréfiant jusqu'au voisinage de la bifurcation intestinale ; limités à la face ventrale du segment postérieur et formant deux amas latéraux posttesticulaires, presque terminaux. EEufs, 84-96/60-68 $\mu$, pouvant atteindre la vingtaine.

HôTES: Melanerpes superciliaris murcens (Bangs) et $M$. superciliaris superciliaris (Temm.).

Anolis equestris kosleri (sic) (10) (hôte accidentel !).

Distribution: Isla de Pinos, Rancho del Tesoro (11-X-1965) [1463, $b, c, d, e$; $1466 a$, de Anolis] ; La Sola Cairije, Sierra Cubitas (26-X-1965) [1484d].

Peninsula de Guanahacabibes, Reservación (25-I-1966) [1933 d].

Holotype : $1463 c$.

Par sa morphologie, la proportion des segments et la distribution des vitellogènes, cette nouvelle espèce se rapproche de Neodiplostomum (N.) travassosi Dubois, 1937, dont elle se distingue par ses dimensions plus élevées, la subégalité des ventouses, le plus grand développement des testicules qui occupent presque tout le segment postérieur, enfin, par la localisation plus avancée des amas folliculaires posttesticulaires, qui n'atteignent pas tout à fait l'extrémité du corps. La structure de la bourse copulatrice n'a pas pu être étudiée.

\section{Neodiplostomum (Neodiplostomum) ellipticum ellipticum}

(Brandes, 1888) La Rue, 1926

Syn. Conchogaster ellipticus (Brandes) Lutz, 1928.

La brève mention originale de ce parasite de Cuculidés est basée sur l'examen du matériel que Natterer a récolté au Brésil chez Piaya cayana (L.). Deux préparations de la collection A. Lutz proviennent du même hôte; une troisième est étiquetée «Conchogaster crotophagae ( (cf. Dubois, $1970 \mathrm{~b}$ ). Dubois (1938) observe deux matériels du

(9) En tenant compte de l'aplatissement que favorise la macération.

(10) Le nom subspécifique doit être erroné. Il s'agit très probablement de l'Anolis equestris hassleri Barbour et Schreve, 1935, de 1'Isla de Pinos. 
Musée de Vienne, provenant du Grand Ani, Crotophaga major Gm. Pérez Vigueras (1944) redécrit le parasite d'après un exemplaire recueilli dans Crotophaga ani L. aux environs de La Havane (cf. «Synopsis des Strigeidae et des Diplostomatidae», fig. 513).

L'espèce a été retrouvée à Cuba dans trois Anis, à Playa Larga. Les matériels, assez mal conservés, n'ont permis qu'une observation sommaire (vide infra).

$N$. ellipticum est caractérisé par le galbe largement ovale du segment antérieur, par la massiveté du segment postérieur ellipsoïdal à sacciforme, plus court chez les exemplaires jeunes, de même longueur ou plus long chez les adultes, par la subégalité des ventouses et le contour circulaire ou elliptique (transversalement) de l'organe tribocytique presque centré dans le segment antérieur, par la situation intersegmentaire de l'ovaire ellipsoïdal à réniforme, par des testicules très développés - l'asymétrie du premier, la bilobation du second (dont le plus grand lobe est opposé obliquement au précédent) - , enfin par la profusion des vitellogènes dans le segment antérieur, où ils atteignent le niveau de l'œsophage, du pharynx ou même de la ventouse buccale.

Les quelques mesures suivantes ont été prises sur le matériel $\mathrm{n}^{\circ} 1654$ (dont l'aplatissement rend inutilisables celles qu'on pourrait prendre des segments du corps et des gonades) :

LONGUEUR DES Vers: jusqu'à $1,75 \mathrm{~mm}$. Rapport des longueurs: segment postérieur/ segment antérieur $=0,9-1,6$. Ventouse buccale 60-73/68-89 $\mu$; pharynx 50-68/45-60 $\mu$; ventouse ventrale 65-73/70-75 $\mu$. Eufs $94-110 / 57-68 \mu$, au nombre de 1 à 9 .

\section{HôTE : Crotophaga ani L.}

Distribution: Peninsula de Zapata, Playa Larga (4-XII-1965) [1653 a; $1654 c$, $d, e ; 1655 a, b]$.

Nous rapportons encore à $N$. ellipticum les matériels en général très macérés, trouvés dans deux sous-espèces d'un Cuculidé des Antilles, Saurothera merlini. Sur les moins mauvaises préparations ( $\mathrm{n}^{\circ s} 1423 f, 1431 a, 1838 a$ ), on peut observer l'habitus caractéristique du Ver (qui mesure 1,12-1,44 mm), la profusion des vitellogènes dans le segment antérieur, la subégalité des ventouses, la forme et la topographie des gonades. Les œufs, dont le nombre peut atteindre une vingtaine, ont comme dimensions 89-104/53-69 $\mu$.

Hôtes : Saurothera merlini decolor Bangs et Zappey, $S$. merlini merlini d'Orbigny.

Distribution: Isla de Pinos, La Vega (7-X-1965) [1423 b, $c, d, f],(8-\mathrm{X}-1965)$ [1431 a $]$.

Peninsula de Zapata, St. Tomas, Canal de Zapata (6-XII-1965) [1685 et 1685 ] .

Peninsula de Guanahacabibes, Maria la Gorda (16-I-1966) [1838 a].

Plusieurs exemplaires de $N$. ellipticum - la plupart ovigères (1 à 12 œufs presque tous déformés) - ont été récoltés dans quatre Corbeaux (Corvus nasicus Temm.), la plupart au même endroit et à la même époque que ceux des Anis. Bien entendu, il s'agit ici d'hébergements accidentels, résultant de la cohabitation des hôtes dans un territoire d'habitat, de nidification ou de nourriture. On sait cependant qu'il existe une 
défense interspécifique entre Crotophaga ani et Corvus nasicus, liée peut-être à une compétition écologique (cf. Noël Mayaud: in Traité de Zoologie de Pierre-P. Grassé. t. XV, p. 727).

Les mesures prises sur les quatre matériels (plus ou moins aplatis) mentionnés cidessous sont les suivantes :

LONGUEUR DES Vers : 1,2-1,8 mm. Segment antérieur ovale, cochléariforme, à cuticule spinescente, $0,70-1,12 / 0,74-0,96 \mathrm{~mm}$; segment postérieur ovoïde ou ellipsoïdal à sacciforme, 0,50-0,82/0,52-0,68 mm. Rapport des longueurs: segment postérieur/segment antérieur = $0,6-0,8$. Ventouse buccale 78-102/80-107 $\mu$; pharynx 63-78/42-62 $\mu$; ventouse ventrale 85 108/94-120 $\mu$; organe tribocytique 225-290/225-320 $\mu$. Ovaire 95-140/185-250 $\mu$; testicule antérieur 210-390/240-440 $\mu$; testicule postérieur 160-370 $\mu$ (petit lobe), 250-530 $\mu$ (grand lobe) $/ 450-660 \mu$.

Hôte: Corvus nasicus Temm.

Distribution: Peninsula de Zapata, Playa Larga (4 et 6-XII-1965) [1665 $d$; $1666 a, b ; 1682 a]$.

Peninsula de Guanahacabibes, Reservación (27-I-1966) [1937 a].

\section{Neodiplostomum (Neodiplostomum) isomegalocotyle n. sp.}

Un seul exemplaire, bien conservé mais contracté, a été recueilli dans un Falco sparverius L. Il est bien caractérisé par l'égalité de ses grandes ventouses.

DiAgnose. Longueur du Ver: 1,26 mm. Segment antérieur de contour ovale, 0,68/ $0,71 \mathrm{~mm}$, à cuticule finement spinescente ; segment postérieur largement ellipsoïdal, 0,58 / $0,70 \mathrm{~mm}$, nettement distinct du précédent par une constriction. Rapport des longueurs: segment postérieur/segment antérieur $=0,85$. Ventouses relativement grandes, approximativement de même diamètre : ventouse buccale $115 / 130 \mu$, ventouse ventrale $110 / 148 \mu$; pharynx 78/ $65 \mu$; œsophage court, $28 \mu$; organe tribocytique arrondi, $220 / 260 \mu$.

Ovaire ellipsoïdal, $125 / 180 \mu$, submédian, situé tout au début du segment postérieur. Testicule antérieur asymétriquement développé, $215 / 530 \mu$; testicule postérieur bilobé, 220$245 / 615 \mu$; vésicule séminale grande, localisée entre ses deux lobes. Vitellogènes envahissant le segment antérieur sur toute sa largeur et s'avançant quelque peu au-delà de la ventouse ventrale sous forme de six traînées de follicules; largement répandus à la face ventrale du segment postérieur, surtout dans la zone prétesticulaire, et atteignant l'extrémité du corps. Bourse copulatrice largement ouverte dorsalement. Eufs 102-104/57-67 $\mu$, au nombre de 5 .

Hôte : Falco sparverius L.

Distribution : Peninsula de Guanahacabibes, Maria la Gorda (16-I-1966) [1841 b].

Holotype : $1841 b$.

Trois exemplaires de ce parasite, assez mal conservés, ont été retrouvés dans un Passériforme du genre Quiscalus Vieill. Ils sont bien caractérisés, eux aussi, par l'égalité de leurs deux grandes ventouses. 


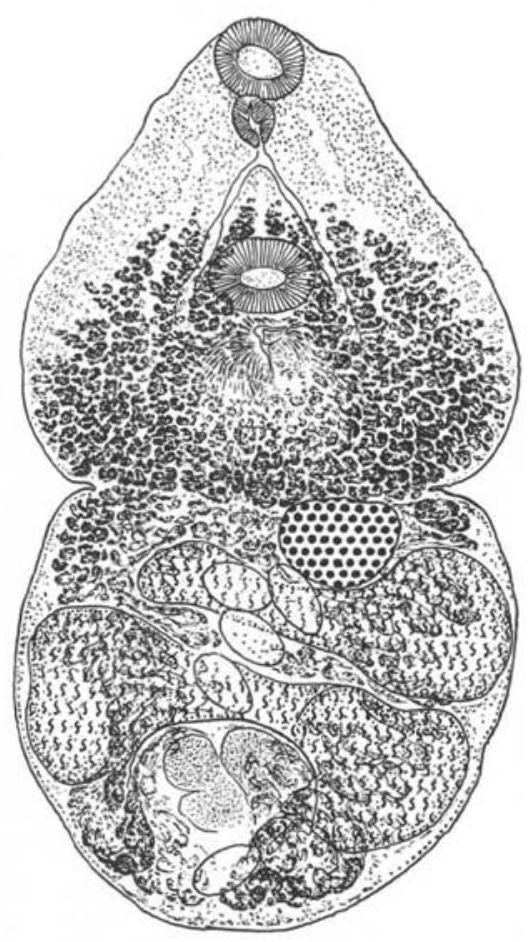

FIG. 7. - Neodiplostomum (Neodiplostomum) isomegalocotyle n. sp., de Falco sparverius L. Holotype. Longueur 1,26 mm, vue dorsale $[1841 \mathrm{~b}]$

LONGUEUR DES VERS : 1,12-1,50 mm. Segment antérieur lancéolé (en extension), 0,75-0,91/ 0,34-0,43 $\mathrm{mm}$; segment postérieur ellipsoïdal, 0,40-0,59/0,25-0,40 mm, nettement distinct du précédent par une constriction. Rapport des longueurs : segment postérieur/segment antérieur $=0,53-0,70$. Ventouses relativement grandes, arrondies et de même diamètre: ventouse buccale $95-105 \mu$; ventouse ventrale $95-105 / 108-110 \mu$; pharynx $80 / 65 \mu$; œsophage court, $40 \mu$; organe tribocytique arrondi, $155-220 / 170-240 \mu$.

Gvaire ellipsoïdal, 70-85/90-130 $\mu$, situé à la limite intersegmentaire ou tout au début du segment postérieur. Testicule antérieur asymétrique, 130-170/170-230 $\mu$; testicule postérieur bilobé, 100-175/190-320 $\mu$. Vitellogènes envahissant le segment antérieur sur toute sa largeur et s'avançant quelque peu au-delà de la ventouse ventrale sous forme de quatre traînées de follicules; largement répandus à la face ventrale du segment postérieur, à peu près jusqu’à l'extrémité du corps. Eufs 100-115/73 $\mu$, au nombre de 2 à 4 .

Hôte : Quiscalus niger caribaeus (Todd).

Distribution: Isla de Pinos, Cayo Piedra (8-X-1965) [1435 $a, e]$. 


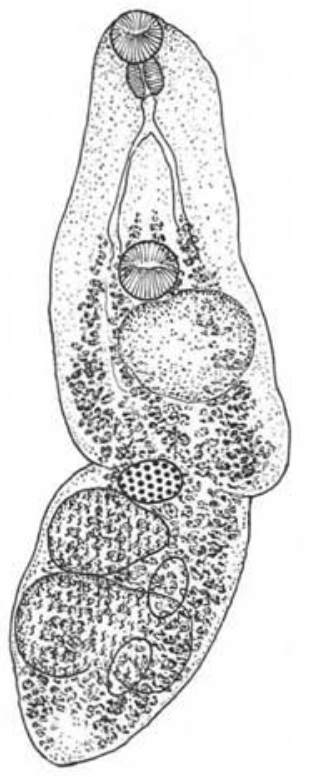

FIG. 8. - Neodiplostomum (Neodiplostomum) isomegalocoty. le n. sp., de Quiscalus niger caribaeus (Todd). Longueur $1,50 \mathrm{~mm}$, vue ventrale (L'organe tribocytique est déformé.) [1435 a ]

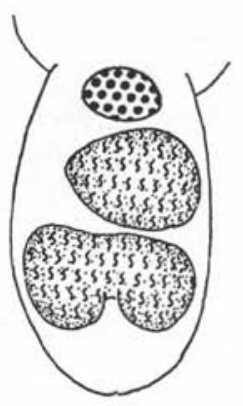

FIG. 9. - Neodiplostomum (Neodiplostomum) isomegalocotyle n. sp., de Quiscalus niger caribaeus (Todd). Morphologie et topographie des gonades [1435 $a$ ]

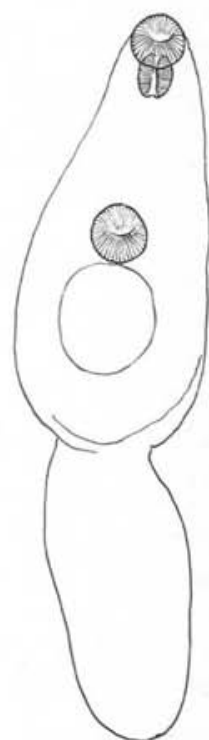

FIG. 10. - Galbe de Neodiplostomum (Neodiplostomum) isomegalocotyle $\mathrm{n}$. $\mathrm{sp}$., de Quiscalus niger ca. ribaeus (Todd). Longueur : $1,34 \mathrm{~mm}$ $[1435 e]$

Posthodiplostomum macrocotyle Dubois, 1937

Cette espèce a été découverte dans l'intestin d'un Rhynchops nigra L., au Brésil. Elle était la seule du genre Posthodiplostomum à n'être pas hébergée par des Ardeae. Or, dans la collection de Cuba, elle se trouve inféodée à plusieurs Oiseaux de ce sousordre, qui en sont probablement les hôtes normaux.

$P$. macrocotyle est caractérisé par son segment antérieur oblong, par la possession d'un acétabulum dont le diamètre moyen peut excéder le double de celui de la ventouse buccale, par la forme en $\mathrm{V}$ ouvert en avant du second testicule dont les deux lobes divergents atteignent les côtés du corps, par la distribution des vitellogènes: dans le segment antérieur, les follicules s'accumulent autour de l'organe tribocytique et se prolongent plus ou moins loin en avant de la ventouse ventrale, tout en diminuant de densité ; dans le segment postérieur, ils forment deux rubans ventraux parallèles, parfois confondus, mais qui divergent peu à peu pour constituer, en arrière des testicules, deux amas latéraux au niveau de la vésicule séminale, laissant à découvert la bourse copulatrice. 
Les mesures que nous avons prises sur les matériels cubains (tous mal conservés) sont les suivantes :

LONGUEUR DU VER : $1 \mathrm{~mm}\left(\mathrm{n}^{\circ} 1643 d\right)$. Segment antérieur long de 0,55-0,77 mm; segment postérieur $0,45-0,52 / 0,22-0,27 \mathrm{~mm}$. Ventouse buccale $28-42 / 28-40 \mu$; pharynx $25-31 / 24-30 \mu$; ventouse ventrale $42-73 / 47-73 \mu$; organe tribocytique $110-180 / 130-170 \mu$. Ovaire $65-70 / 80$ $100 \mu$; testicule antérieur 125-155/135-155 $\mu$; testicule postérieur 165-200/210-225 $\mu$. CEufs 80-90/50-60 $\mu$, au nombre de 2 ou 3. Situation de la ventouse ventrale dans le segment antérieur: 60-65/100. Longueur des groupes latéro-subterminaux de vitellogènes : 105-140 $\mu$.

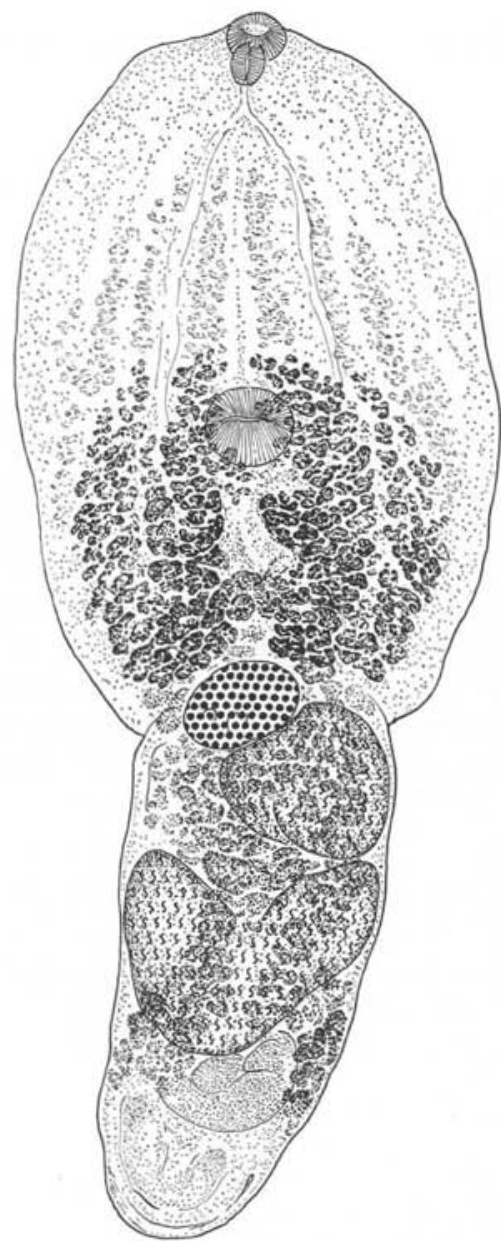

FIG 11. - Posthodiplostomum macrocotyle Dubois, de Butorides virescens maculatus (Bodd.). Longueur: $1 \mathrm{~mm}$, vue dorsale $[1643 d]$ 
Hôtes : Florida caerulea (L.), Egretta alba (L.), Egretta alba egretta (Gm.), Hydranassa tricolor ruficollis (Gosse), Nycticorax nycticorax hoactli (Gm.), Butorides virescens maculatus (Bodd.) $=$ B. brunescens.

Distribution : Isla de Pinos, Camino de San Francisco (8-X-1965) [1442 b, $d, e]$; Canal de Vigil, La Gloria (28-X-1965) [1490 $f, i]$.

Peninsula de Zapata, St. Tomas, Canal de Zapata (2-XII-1965) [1633 b], (1-XII1965) $[1639 e, 1642,1643 d, e]$.

Posthodiplostomum nanum Dubois, 1937

Syn. Posthodiplostomum antillanum Pérez Vigueras, 1944.

Cette petite espèce brésilienne, trouvée maintes fois dans la collection A. Lutz (cf. Dubois 1970 b), a été redécrite par Pérez Vigueras (1944) sous le nom de Posthodiplostomum antillanum d'après quelques exemplaires recueillis chez un Butorides virescens maculatus (Bodd.). La description et la figure 13 de cet auteur révèlent bien les caractéristiques du stade adulte, à savoir :

Segment antérieur arrondi ou largement ovale, à concavité ventrale accusée par le rebord latéro-postérieur, délimité par une constriction du second segment typiquement ovoïde, plus court et plus étroit. Ventouses subégales : acétabulum à peine plus grand que la buccale et proche de l'organe tribocytique arrondi ; glande protéolytique développée en largeur au niveau de son bord caudal, bilobée et échancrée médio-postérieurement. Ovaire ellipsoïdal, submédian ; testicule antérieur asymétrique, ovoïde et latéral ; second testicule massif, subréniforme, à convexité postérieure. Vitellogènes amplement distribués dans le segment antérieur, jusqu'au-devant de l'acétabulum, formant deux petits amas de follicules au début du segment postérieur, puis raréfiés sur toute la face ventrale et se terminant au niveau du bord caudal du second testicule. Eufs très rares (un ou deux).

LONGUEUR DU Ver : 0,68-0,97 mm. Segment antérieur 0,36-0,58/0,34-0,53 mm; segment postérieur $0,32-0,40 / 0,26-0,39 \mathrm{~mm}$. Rapport des longueurs : segment postérieur/segment antérieur $=0,55-0,90$. Ventouse buccale $35-57 / 31-50 \mu$; ventouse ventrale $37-55 / 42-57 \mu$; pharynx $30-50 / 21-40 \mu$; organe tribocytique $80-140 / 100-140 \mu$; glande protéolytique $52-75 / 140$ $175 \mu$. Ovaire 60-72/75-100 $\mu$; testicule antérieur 110-125/120-130 $\mu$; testicule postérieur $115-140 / 150-210 \mu$.

Hôtes: Florida caerulea (L.), Egretta alba (L.), Nycticorax nycticorax hoactli (Gm.), Leucophoyx thula (Molina).

Distribution: Isla de Pinos, Camino de San Francisco (8-X-1965) [1442e].

Peninsula de Zapata, St. Thomas, Canal de Zapata (1 et 2-XII-1965) [1633 $b$, $1636 c, 1640 \mathrm{fl}$. 


\section{Considérations sur la faune helminthologique de Cuba dans ses relations avec l'avifaune}

L'avifaune des Antilles est pauvre comparativement aux faunes continentales voisines. Cette pauvreté relative se répercute sur l'helminthofaune de Cuba: dans cette île, les Strigeata ne comptent actuellement que 11 genres et 21 espèces (11), qu'on peut répartir en trois groupes :

a) Parmi ces espèces, les unes sont d'origine néocontinentale, venant des régions holarctique ou sonorienne [Apatemon (Apatemon) gracilis (Rudolphi), Apharyngostrigea cornu (Zeder), A. multiovata (Viguèras), Parastrigea mexicana Coil, Cardiocephaloides medioconiger (Dubois et Vigueras), Posthodiplostomum minimum (MacCallum)].

b) D'autres espèces sont néotropicales [Strigea falconis brasiliana Szidat, S. vaginata (Brandes), Parastrigea cincta (Brandes), Cotylurus gallinulae gallinulae (Lutz), Diplostomum (Austrodiplostomum) compactum (Lutz), Diplostomum (Tylodelphys) elongatum (Lutz), Neodiplostomum (Neodiplostomum) ellipticum ellipticum (Brandes), Posthodiplostomum macrocotyle Dubois, $P$. nanum Dubois] ; elles représentent des éléments particuliers au continent sudaméricain, contribuant à accentuer l'originalité faunique de ce dernier.

Parmi les groupes aviens, vecteurs de cette faune strigéidienne typiquement néotropicale, figurent les Vulturidés (Cathartes, Coragyps et Sarcorhamphus pour Strigea vaginata), les Cuculidés (Saurothera, Crotophaga et Piaya pour Neodiplostomum ellipticum) et deux Anatidés [Cairina moschata (L.) et Amazonetta brasiliensis (Gm.), hôtes d'Apatemon (Australapatemon) bdellocystis (Lutz), du Brésil et du Venezuela].

D’autres groupes aviens plus ubiquistes assurent la dispersion de Diplostomidés non moins caractéristiques de cette faune: ce sont, par exemple, les Ardéiformes, vecteurs de plusieurs espèces de Posthodiplostomum (notamment $P$. nanum et $P$. macrocotyle, tous deux fréquents à Cuba), les Phalacrocoracidés ( $P$. olivaceus et $P$. auritus) qui hébergent le Diplostomum (Austrodiplostomum) compactum, et les Piciformes dont les genres Melanerpes et Rhamphastos étaient porteurs de Neodiplostomum centuri n. sp. et de $N$. travassosi Dub., respectivement.

Le peuplement avien néotropical est altéré dans son originalité par l'intrusion de quelques types génériques véritablement cosmopolites, tels que les Grèbes (Podiceps et Podilymbus), hôtes de Diplostomum (Tylodelphys) elongatum (Lutz) (12), les Rapaces diurnes et nocturnes (Buteo, Falco; Asio, Bubo, Strix), auxquels s'ajoutent deux Echasses (Himantopus et Recurvirostra), vectrices de Parastrigea mexicana.

c) Quelques espèces de Strigeata seulement pourraient être indigènes, propres à la faune cubaine, entre autres Schwartzitrema schwartzi (Vigueras), de Anhinga anhinga (L.), jamais retrouvé ailleurs, Neodiplostomum morenodathei Odening, 1970, d'une Chouette de Cuba, Gymnoglaux lawrenci exul (Bangs) (13), et Neodiplostomum centuri n. sp., dont l'hôte est un des Melanerpes abondants surtout dans les Antilles et en Amérique centrale.

(11) D'après les travaux de Pérez Vigueras $(1944,1955)$, d'Odening $(1970 a, b)$ et la présente étude.

(12) Ce Diplostome a été trouvé au Venezuela et à Cuba.

(13) Le spécimen obtenu d'Asio flammeus, qu'Odening représente (1970 $b$, fig. $5 f$ ) et considère comme un «fragliches Exemplar», nous paraît identifiable avec Neodiplostomum spathoides Dub. L'hôte, importé du Canada le 28 juillet 1963, a été autopsié le 30 octobre : il a dû s'infester durant sa captivité au zoo de Berlin. 
Le caractère composite de la faune helminthologique cubaine exprime d'une manière assez suggestive le mode du peuplement avien dans le Nouveau Monde et peutêtre quelques modalités des migrations continentale: Actuellement, dans le continent nord-américain, la faune néotropicale se propage bien au-delà de l'isthme de Tehuantepec, le long des côtes du Mexique d'une part, dans les Antilles d'autre part, régions où persiste le biotope des forêts tropicales. Inversement, certains éléments de la faune cosmopolite d'origine paléocontinentale (qui a envahi par le nord le nouveau continent) et de la faune sonorienne essaiment ou effectuent des déplacements migratoires jusqu'en Amérique centrale et dans l'archipel antillien. C'est ainsi que la distribution géographique de Cardiocephaloides medioconiger (le long de la côte Atlantique, du Maine jusqu'en Floride, dans le golfe du Mexique jusqu'à Panama, à Cuba et à Porto-Rico) jalonne une voie de migration d'un des Laridés (Thalasseus maximus, hôte de ce parasite); que celle d'Apharyngostrigea multiovata (comprenant le Wisconsin, l'Ohio, les grandes Antilles et le Brésil) suit probablement un des itinéraires utilisés par des Ardéidés hébergeant ce Strigéide.

\section{Bibliographie}

BeVERley-Burton (M.), 1963. - A new Strigeid, Diplostomum (Tylodelphys) mashonense n. sp. (Trematoda: Diplostomatidae), from the Grey Heron, Ardea cinerea L. in Southern Rhodesia, with an experimenal demonstration of part of the life cycle. Rev. Zool. Bot. Afr., LXVIII, 291-308.

Brandes (G.), 1888. - Die Familie der Holostomeae. Ein Prodromus zu einer Monographie derselben (Inaug.-Diss.), 72 pp., Reudnitz, Leipzig.

-, 1890. - Die Familie der Holostomiden. Zool. Jahrb. (Syst.), V, 549-604.

BYRD (E. E.) et WARD (J. W.), 1943. - Notes on the genital system of the bird fluke, Apharyngostrigea cornu (Zeder). J. Parasit., XXIX, 270-274.

Caballero (E.) et Vogelsang (E. G.), 1949. - Fauna helmintologica venezolana. II. Algunos trematodos de aves y mamiferos. Rev. Med. Vet. y Paras., Caracas, VIII, 1-23.

Cable (R. M.), Connor (R. S.) et Balling (J. W.), 1960. - Digenetic trematodes of Puerto Rican shore birds. Scient. Survey Porto Rico, XVII, 187-255.

Coll (W. H.), 1957. - Parastrigea mexicanus sp. nov., a strigeid trematode from the avocet. Trans. Amer. micros. Soc., LXXVI, 70-72.

Dubors (G.), 1938. — Monographie des Strigeida (Trematoda). Mém. Soc. neuchâtel. Sci. nat., VI, $1-535$.

—, 1958. - Les Strigeida (Trematoda) de Californie de la collection June Mahon. Bull. Soc. neuchâtel. Sci. nat., LXXXI, 69-78.

—, 1967. — Notes helminthologiques. I. Strigeidae Railliet (Trematoda). Rev. suisse Zool., LXXIV, 693-700.

—, 1968 a. - Du statut de quelques Strigeata La Rue, 1926 (Trematoda). III. Bull. Soc. neuchâtel. Sci. nat., XCI, 5-19.

—, 1968 b. - Synopsis des Strigeidae et des Diplostomatidae (Trematoda). Mém. Soc. neuchâtel. Sci. nat., X (1), 1-258. 
-, 1969. - Les Strigeata (Trematoda) de la collection Elisabeth M. Boyd. Bull. Soc. neuchâtel. Sci. nat., XCII, 5-12.

—, 1970 a. - Synopsis des Strigeidae et des Diplostomatidae (Trematoda). Mém. Soc. neuchâtel. Sci. nat., X (2), 259-727.

—, 1970 b. - Les Strigeata (Trematoda) de la collection A. Lutz. Mém. Inst. Oswaldo Cruz, Rio de J., LXVIII, 169-196.

Dubois (G.) et Pérez Vigueras (I.), 1949. - Notas rectificativas sobre algunos Estrigeideos de la isla de Cuba. Rev. Univ. La Habana $\mathrm{N}^{\circ s} 82-87,260-266$.

Lutz (A.), 1928. - Estudios de Zoologia y Parasitologia Venezolanas. 133 p., Rio de Janeiro.

Mayaud (N.), 1950. - Comportement et vie sociale. In: Traité de Zoologie de Pierre-P. Grassé, t. XV (Oiseaux), 697-746, Paris (Masson et $\mathrm{C}^{i e}$ ).

Odening (K.), 1970 a. - Eine neue Neodiplostomum-Art (Trematoda) aus einem Kubakauz. Zool. Anz., CLXXXIV, 121-124.

—, 1970 b. - Neue Funde von Diplostomidae (Trematoda) aus Vögeln des Berliner Tierparks Mitt. Zool. Mus. Berlin, XLVI, 167-181.

OLSEN (O.W.), 1940. - Two new species of trematodes (Apharyngostrigea bilobata: Strigeidae, and Cathaemasia nycticoracis: Echinostomidae) from Herons, with a note on the occurence of Clinostomum campanulatum (Rud.). Zoologica, N. Y., XXV, 323-328.

Pérez Vigueras (I.), 1944. - Trematodes de la super-familia Strigeoidea; descripcion de un genero y siete especies nuevas. Rev. Univ. La Habana $\mathrm{N}^{\circ s}$ 52-54, 293-314.

-, 1955. - Contribucion al conocimiento de la fauna helmintologica cubana. Mem. Soc. cubana Hist. nat., XXII, 195-233.

Szidat (L.), 1929. - Beiträge zur Kenntnis der Gattung Strigea (Abildg.). II. Spezieller Teil : Revision der Gattung Strigea nebts Beschreibung einer Anzahl neuer Gattungen und Arten. Z. Parasitenk., I, 688-764.

Szidat (L.) et NANI (A.), 1951. - Diplostamiasis cerebralis del pejerrey. Una grave epizootia que affecta a la economia nacional producida por larvas de Trematodes que destruyen el cerebro de los pejerreyes. Rev. Mus, arg. Cienc. nat. Bernard Rivad. (Cienc. Zool.), I, 323-384. 